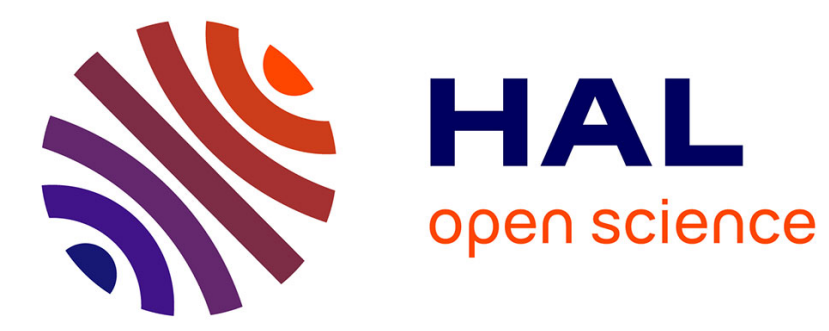

\title{
Optimal Motion Generation for Mobile Robot with Non-Skidding Constraint
}

\author{
Jiuchun Gao, Anatol Pashkevich, Fabien Claveau, Philippe Chevrel
}

\section{To cite this version:}

Jiuchun Gao, Anatol Pashkevich, Fabien Claveau, Philippe Chevrel. Optimal Motion Generation for Mobile Robot with Non-Skidding Constraint. 2019 IEEE International Conference on Mechatronics (ICM), Mar 2019, Ilmenau, Germany. pp.628-633, 10.1109/ICMECH.2019.8722896 . hal-02148787

\section{HAL Id: hal-02148787 https://hal.science/hal-02148787}

Submitted on 14 Jun 2019

HAL is a multi-disciplinary open access archive for the deposit and dissemination of scientific research documents, whether they are published or not. The documents may come from teaching and research institutions in France or abroad, or from public or private research centers.
L'archive ouverte pluridisciplinaire HAL, est destinée au dépôt et à la diffusion de documents scientifiques de niveau recherche, publiés ou non, émanant des établissements d'enseignement et de recherche français ou étrangers, des laboratoires publics ou privés. 


\section{Optimal motion generation for mobile robot with non-skidding constraint}

\author{
Jiuchun Gao \\ The laboratory of Digital \\ Sciences of Nantes (LS2N) \\ Institute Mines-Telecom \\ Atlantique \\ Nantes, France \\ jiuchun.gao@imt-atlantique.fr
}

\author{
Anatol Pashkevich \\ The laboratory of Digital \\ Sciences of Nantes (LS2N) \\ Institute Mines-Telecom \\ Atlantique \\ Nantes, France \\ anatol.pashkevich@imt- \\ atlantique.fr
}

\author{
Fabien Claveau \\ The laboratory of Digital \\ Sciences of Nantes (LS2N) \\ Institute Mines-Telecom \\ Atlantique \\ Nantes, France \\ fabien.claveau@imt-atlantique.fr
}

\author{
Philippe Chevrel \\ The laboratory of Digital \\ Sciences of Nantes (LS2N) \\ Institute Mines-Telecom \\ Atlantique \\ Nantes, France \\ philippe.chevrel@imt- \\ atlantique.fr
}

\begin{abstract}
The paper presents a new motion generation technique for a mobile robot that is able to find time-optimal motions along a curved path that takes into account limitations of the driving motors and also ensures the wheels rolling without skidding. Mathematically, the problem is presented as a timeoptimal control of a second order dynamic system under constraints on the control input, the first derivative of output, and also mixed constraint on the control variable and the output derivative. After the state space discretization, the original problem is converted into the combinatorial one where the desired robot trajectory is presented a shortest path on the relevant graph. To find this path and ensure its physical implementation, dynamic programming is applied that allows creating a set of feasible trajectories, the best one of which is selected at the final stage. Advantages of the proposed technique and its suitability of real-time control are illustrated by several examples dealing the mobile robot motion along the elliptic and star-type paths.
\end{abstract}

Keywords-mobile robot, motion generation, time-optimal control, non-skidding constraint

\section{INTRODUCTION}

In the few last years, due to important developments in computer vision and computational geometry, it has been achieved essential progress in the area of mobile robot path planning. Existing on-board robot controllers are able to generate the path in real-time for very complex, clustered and partially unknown environment. At the same time, the problem of time-optimal motion along the obtained path received less attention; existing techniques mainly rely on the classical phase-plane methods that require some non-trivial actions in the case of complex state-dependent constraints. On the other side, the problem of time-optimal motion along a specified path has been intensively studied in robotics for several decades. In early works, the authors concentrated on serial robotic manipulators and the desired optimal motions of the robot end-effector were generated taking into account the velocity/acceleration bounds for the joint variables. The latter is consistent with physical limitations of the actuating motors, which have obvious limits on the maximum rotation speeds and peak motor torques. Among the key contributions in this area are the papers of Bobrow et al. [1]; Pfeiffer and Johanni [2]; Shiller et Lu [3, 4]; Shin and McKay [5]; and Slotine and Yang $[6,7]$. These works employ the phase-plane method and rely on the fact that, generally, the time-optimal solution is "bang-bang", i.e. at each time instant one of the actuators operates at the limit of its speed or acceleration. Because of the geometric constraints imposed by the given path, the state vector includes here only two variables that are the distance and velocity along the path. However, the non-linear manipulator dynamics and kinematics transforms the actuators constraints into state-dependent constraints on velocity and acceleration along the path. Thus, the original problem is reduced to the time-optimal control of a second-order linear system with nonlinear state-dependent constrains on the velocity and acceleration, where the acceleration is also treated as the control input. In the above mentioned works, the authors have presented a number of interesting examples and detected some important properties of the time-optimal control (such as multiple switchings between the acceleration and deceleration), which are not common for the second-order dynamic systems studied in the classical control theory. Nevertheless, in the frame of the phase-plane method, no any general technique was developed that allows generate the multiple-switching optimal control for the considered problem.

Among other related contributions, it worth mentioning several works dealing with time-optimal control of multi-axes CNC machines where it is necessary to specify the feedrate variation along a curved path yielding minimum traversal time subject to prescribed acceleration bounds along each axis. Here, the optimal solution is also based on the "bang-bang", principle, i.e. the maximum acceleration/deceleration is applied at least at one axis at each time instant, but with the switching points were computed from the roots of certain polynomials [8]. More resent results focuses on the feedrate optimization taking into account high-order constraints (i.e. axes velocities, accelerations, jerks, etc.) $[9,10]$. However, there is an essential practical difficulty in this application area that is caused by common industrial practice of approximating free-form curved paths by short linear/circular G-code segments, which makes the realization of continuously-varying feedrates problematic. This difficulty is gradually disappearing in new CNC machines that allow the real-time interpolation for analytic curves, which include the path curvature and the feedrate variation.

For mobile robots, the time-optimal control problem is usually stated in a slightly different way, assuming that the path is not given in advance. It is generated at the first stage 
using relevant geometric algorithms and taking into account constraints imposed by workspace obstacles, required path smoothness and nonholonomy of the mobile robot $[11,12]$. Then at the second stage, a time-optimal velocity profile is generated under dynamic constraints that are due to the motor physical capacities and also must ensure the wheel rolling without skidding [13]. The non-skidding condition can be presented as a non-linear constraint imposed on the velocity and acceleration, which leads to the similar problem as for the robotic manipulators (with multiple switchings of optimal control). In most of existing works, the desired time-optimal profiles are obtained using the phase-plane method, which in the case of multiple switchings is difficult for algorithmization and can be hardly implemented in mobile robot controllers. For this reason, this work concentrates on development of a universal technique and relevant algorithms that allow generating the time-optimal motions for any combinations of linear/nonlinear constraints on velocity and acceleration and is fast enough to be suitable for real-time implementation.

\section{PROBLEM STATEMENT}

Let us assume that the desired path of the mobile robot is presented in the parametric form

$$
\left\{x(s), y(s) ; s \in\left[0, s_{\max }\right]\right\}
$$

describing evolution of Cartesian coordinates of the reference point $(x, y)$ while moving along the given path where $\mathrm{s}$ the distance from the origin to the current state (treated here as the parameter). In addition, let us define the path curvature described by the radius function

$$
r(s), s \in\left[0, s_{\max }\right]
$$

which can be computed from $x(s), y(s)$ using standard formulas. The problem is to find the motion law along the path $s(t), t \in\left[0, t_{\max }\right]$ that ensures the minimum travelling time from the initial state $s=0$ to the final state

$$
t_{\max } \rightarrow \min
$$

and satisfies certain constraints on the derivatives of $s(t)$

$$
|\dot{s}(t)| \leq v_{\max } ; \quad|\ddot{s}(t)| \leq a_{\max } ; \quad g(\dot{s}(t), \ddot{s}(t)) \leq c_{\max }
$$

The first two of the above constraints describe physical limitations of the robot driving motors. The third constraint takes into account physical properties of the contact "robot wheel - road surface" where the total inertia force cannot exceed the friction force. It can be easily proved that after some simplifications the latter condition can be presented in the following form

$$
\left(\frac{\dot{s}(t)^{2}}{r(t)}\right)^{2}+\ddot{s}(t)^{2} \leq(\mu \cdot g)^{2}
$$

where the components on the left-hand side are the lateral and longitudinal accelerations respectively. $g$ is the acceleration of gravity, and $\mu$ is the friction coefficient.

Using terminology from the optimal control theory and considering the acceleration as a control input, the considered problem can be present as minimization of the cost functional

$$
\int_{0}^{T} 1 \cdot d t \rightarrow \min
$$

subject to the second-order dynamic constraints

$$
\ddot{s}=u
$$

with boundary conditions $s(0)=0 ; s(T)=s_{\max } ; \dot{s}(0)=0$; $\dot{s}(T)=0$ and the algebraic constraints on the control variable and one of the state variables

$$
|u| \leq a_{\max } \quad ; \quad|\dot{s}| \leq v_{\max }
$$

as well as a mixed constraint imposed on the state variable and control (defines the limit of centrifugal forces also)

$$
\left(\frac{\dot{s}^{2}}{r(s)}\right)^{2}+u^{2} \leq u_{0}^{2}
$$

where the parameter $u_{0}=\mu g$ denotes the maximum acceleration bound from the friction. The latter also gives some upper bounds for the state variable and control, such as $|u| \leq u_{0}$ and $|\dot{s}| \leq \sqrt{u_{0} \cdot r(s)}$ that may replace the previous ones.

If the last of the above constraints is not active, the optimal solution is trivial and can be easily obtained using previously developed methods. In this case the desired motion is obtained by sequentially applying accelerations $\left(+a_{\max }, 0,-a_{\max }\right)$ that produce either the trapezoid or triangular velocity profile. However, in general case, the constraints may compete to each other and the structure of the optimal solution becomes more complicated, which creates essential difficulties with the optimal motion generation for real-time applications.

\section{OPTIMAL MOTION GENERATION UsING ThE PHASE PLANE TECHNIQUE}

In classical control theory the time-optimal motions for the second-order dynamic systems are usually generated using the phase-plane technique, taking into account specific properties of the optimal control input $u(t)$. It was strictly proved that in the simplest case, when only the input constraint $|u| \leq a_{\max }$ is applied to the dynamic system (7), the optimal control has a "bang-bang" structure, where the amplitude is constant $|u(t)|=a_{\max }$ and there is a single switching point from $u=+a_{\max }$ to $u=-a_{\max }$. For the more complex case, where 
both constraints $|u| \leq a_{\max }$ and $|\dot{s}| \leq v_{\max }$ are taken into account, the optimal control is also discontinuous but it includes an intermediate interval with $u(t)=0$ ensuring satisfaction of the velocity constraint. So, here there are two switchings, from $u=+a_{\max }$ to $u=0$ and from $u=0$ to $u=-a_{\max }$. However, as follows from relevant studies, application of the additional non-linear constraint (9) can crucially change the structure of the optimal control function $u(t)$, which may include multiple switchings between $\pm a_{\max }$ and 0 [1]. Nevertheless, in some cases, the classical phase-plane technique may be efficient.

Let us present first some modification of the conventional phase-plane technique that allows us to take into account approximately the non-linear constraint (10) assuming that at each time-instant the time-optimal control operates at the limit of either of the control input $u$ or the admissible velocity $\dot{s}$. A basis idea here is to replace the primary upper bounds on the velocity and acceleration (8) by new values depending on the current state of the dynamic system $(s, \dot{s})$. Such way of combination of the inequalities (8) and (9) yields the modified bounds of the control input $u$ and the velocity $\dot{s}$ :

$$
\begin{aligned}
& \hat{a}_{\text {max }}=\min \left\{a_{\text {max }}, \sqrt{u_{0}^{2}-\left(\dot{s}^{2} / r(s)\right)^{2}}\right\} \\
& \hat{v}_{\text {max }}=\min \left\{v_{\text {max }}, \sqrt{u_{0} r(s)}\right\}
\end{aligned}
$$

that are included in the time-optimal motion planning algorithm in the following way:

(1) Integrate forwards (increasing $s)$ from $(0,0)$ to $\left(s_{\max }, \dot{s}\right)$ in the phase-plane $(s, \dot{s})$ by applying: (i) the maximum control input $u=\hat{a}_{\max }$ if the velocity constraint $|\dot{s}| \leq \hat{v}_{\max }$ is satisfied, or (ii) zero control input $u=0$ otherwise.

(2) Integrate backwards (decreasing $s)$ from $\left(s_{\max }, \dot{s}\right)$ to $(0,0)$ by applying: (i) the minimum control input $u=-\hat{a}_{\max }$ if the velocity constraint $|\dot{s}| \leq \hat{v}_{\max }$ is satisfied, or (ii) zero control input $u=0$ otherwise.

(3) Find the meeting point of the forward and backward trajectories, and join them at the intersection point.

It worth mentioning that the above algorithm works perfectly well if the non-linear constraint (10) is not too hard and it is satisfied even for the trajectory segments corresponding to $u=0$. However, in the general case, the constraints may compete strongly and the obtained forward and backward trajectories are not feasible. The latter is illustrated by several examples presented in Fig. 1 that demonstrate the motion planning results for several elliptic trajectories $x=a \cdot \cos (\varphi) ; y=b \cdot \sin (\varphi) ; \varphi \in[0,2 \pi]$ with different values of the parameters $a, b$. Here, the system parameters were assumed to be the following $v_{\max }=15 \mathrm{~m} / \mathrm{s}, a_{\max }=5 \mathrm{~m} / \mathrm{s}^{2}$, $\mu=0.7$ which correspond to $u_{0}=6.9 \mathrm{~m} / \mathrm{s}^{2}$. As follows from these results, in the cases (a) and (b) the above presented phase plane technique produces feasible results since the nonskidding constraint (9) is satisfied for all sections of both the forward and the backward trajectories. However, in the case (c) with higher curvature radius, this technique becomes extremely tedious and the obtained trajectories may enter the forbidden region where the non-skidding constraint is violated and the obtained motion is not feasible [14]. The latter motivates us to develop another technique that is capable to overcome this difficulty.

(a)

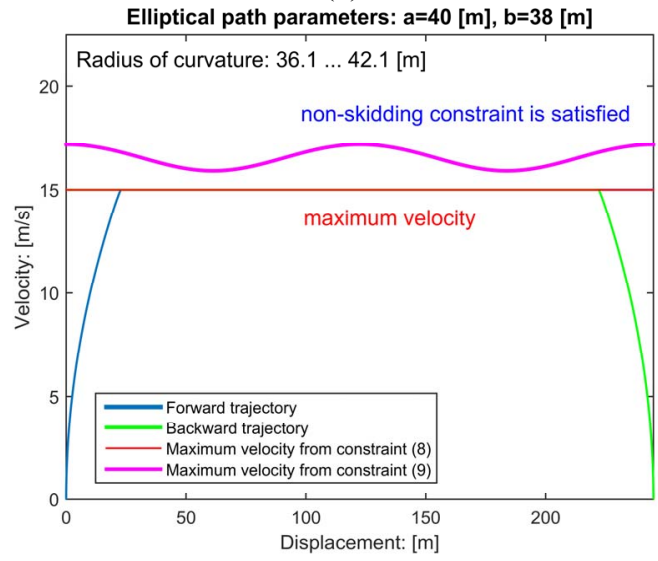

(b)

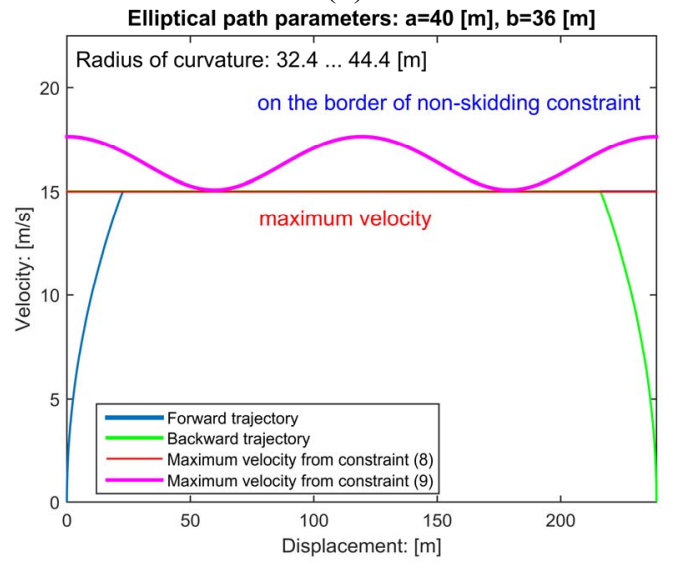

(c)

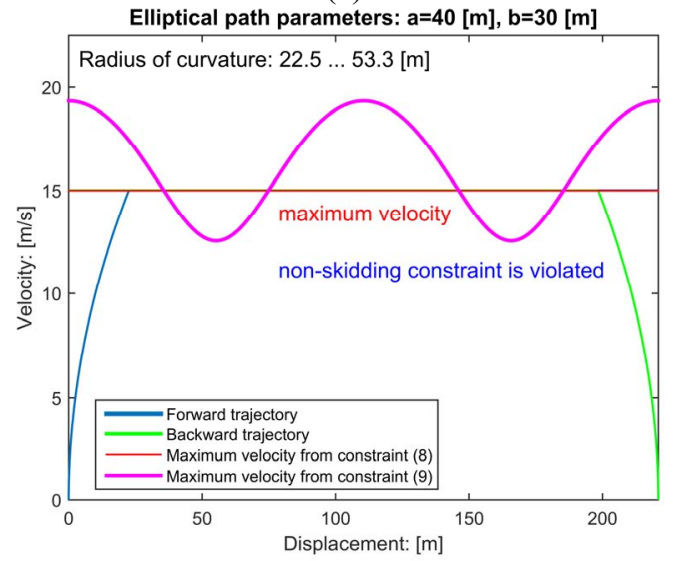

Fig. 1 Motion generated for elliptical path with different curvatures using phase-plane technique 


\section{MOTION GENERATION USING DISCRETE DYNAMIC RROGRAMMING}

An alternative approach to the time-optimal motion generation, which is proposed in this paper, is based on the discrete dynamic programming. Because of its universal nature, this technique is able to take into account all considered constrains in a similar way and to generate optimal trajectories of complex structure that include multiple segments separated by so-called switching points (also known as discontinuity points, tangent points and singular critical points [8]). In contrast to the phase-plane technique, the dynamic programming includes only forward integration but, at each time instant, it considers a set of feasible trajectories from which the best one is selected at the final stage. Let us present a new technique in detail.

\section{A. Search Space Discretization}

To present the problem in a discrete way, let us sample the allowable domain of the velocity and displacement $v \in\left[0, v_{\max }\right]$ and $s \in\left[0, s_{\max }\right]$ with the steps $\Delta v$ and $\Delta s$

$$
\begin{array}{ll}
v_{(k)}=k \cdot \Delta v ; & k=0,1, \ldots m \\
s_{(i)}=i \cdot \Delta s ; & i=0,1, \ldots n
\end{array}
$$

where $\Delta v=v_{\max } / m$ and $\Delta s=s_{\max } / n$. Then, for each path point we can generate a number of the mobile robot states with all possible velocities, i.e. $s_{i} \rightarrow \mathbf{C}_{(k, i)}=\left(v_{(k)}, s_{(i)}\right) ; \forall k$.

While considering the path points are ordered in time, the original sequence of $s_{i}$ described by presented equations may be converted into a directed graph presented in Fig. 2. It should be noted that some of the states generated by previous equation should be excluded from the graph. Here, it is assumed that the velocities at the starting and end points are zero, and for this reason the non-zero velocity states of first and last columns in the graph are inadmissible. These inadmissible states are not connected to any neighbor. It is clear that due to timeirreversibility, the allowable connections between the graph nodes are limited to the subsequent configuration states $\mathbf{C}_{\left(k_{i}, i\right)} \rightarrow \mathbf{C}_{\left(k_{i+1}, i+1\right)}$, and the edge weights correspond to the minimum travelling time.

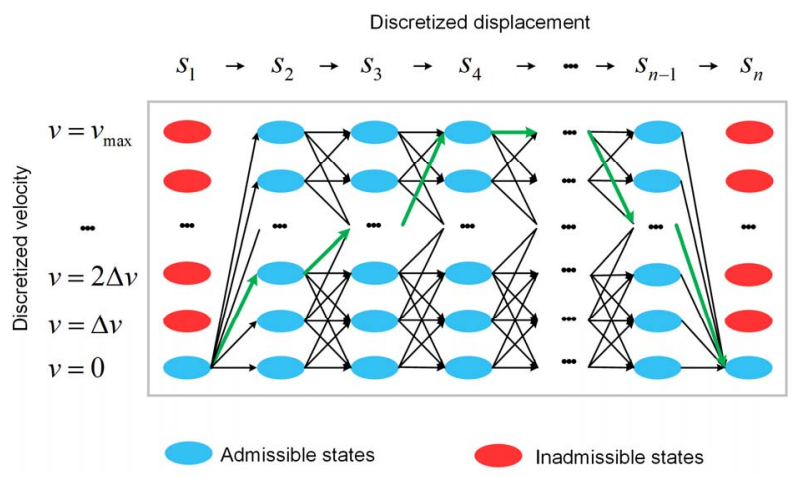

Fig. 2 Graph-based presentation of the discrete search space
Using the discrete search space, the considered problem can be transformed to the searching of the shortest path on the above presented graph. In accordance with the assumption in this work, the initial and final nodes are $\mathbf{C}_{(0,1)}$ and $\mathbf{C}_{(0, n)}$ respectively. In the frame of this notation, the desired solution can be represented as the sequence $\mathbf{C}_{(0,1)} \rightarrow\left\{\mathbf{C}_{\left(k_{2}, 2\right)}\right\} \rightarrow \ldots\left\{\mathbf{C}_{\left(k_{n-1}, n-1\right)}\right\} \rightarrow \mathbf{C}_{(0, n)}$

The distance between subsequent nodes can be evaluated as the displacement time in the following way:

$$
\operatorname{dist}\left(\mathbf{C}_{\left(k_{i}, i\right)}, \mathbf{C}_{\left(k_{i+1}, i+1\right)}\right)=2\left(s_{i+1}-s_{i}\right) /\left(v_{k_{i+1}}+v_{k_{i}}\right)
$$

The latter allows us to present the objective function (travelling time) as follows

$$
T=\sum_{i=1}^{n-1} \operatorname{dist}\left(\mathbf{C}_{\left(k_{i}, i\right)}, \mathbf{C}_{\left(k_{i+1}, i+1\right)}\right)
$$

that depends on the indices $k_{1}, k_{2}, \ldots k_{n-1}, k_{n}$. In this work, it is clear that $k_{1}=k_{n}=0$ when assuming velocities at starting and end points are both zero. It should be noted that the applied method of search space discretization automatically takes into account the velocity constraints, but the acceleration constraints must be examined as follows

$$
\left|\left(v_{i+1}-v_{i}\right) / \Delta t\right| \leq a_{\max }
$$

where $\Delta t=\operatorname{dist}\left(\mathbf{C}_{\left(k_{i}, i\right)}, \mathbf{C}_{\left(k_{i+1}, i+1\right)}\right)$. In addition, the non-skidding condition (5) should be also verified for computing the edge weights as follows

$$
\sqrt{a^{2}+\left(v_{i}^{2} / R_{i}\right)^{2}} \leq \mu \cdot g
$$

where $R_{i}$ is the radius of curvature corresponding to the $i^{\text {th }}$ path point, and $a$ can be estimated from (14).

\section{B. Dynamic Programming Based Technique}

After discretization, the original optimization problem is converted to a combinatorial one, which can generally be transformed to the classical shortest path search on the graph. However, this straightforward approach is extremely timeconsuming compared to dynamic programming based technique [15].

The developed algorithm breaks down the full-size problem into a set of sub-problems, aiming at finding the shortest path from the initial node $\mathbf{C}_{(0,1)}$ to the current $\left\{\mathbf{C}_{\left(k_{i}, i\right)}, \forall k_{i}\right\}$. To present the basic idea, let us denote $d_{k, i}$ as the length of the shortest path connecting the initial node to the current node $\left\{\mathbf{C}_{\left(k^{\prime}, i\right)}\right\}$. Then, taking into account the additivity of the objective (4), the shortest path for the nodes belong to the next layer $\left\{\mathbf{C}_{(k, i+1)}, \forall k\right\}$ can be found by combining the optimal solutions for the previous layer $\left\{\mathbf{C}_{\left(k^{\prime}, i\right)}, \forall k^{\prime}\right\}$ and the distances 
between the nodes with the indices $i$ and $i+1$. The latter corresponds to the formula

$$
d_{k, i+1}=\min _{k^{\prime}}\left\{d_{k^{\prime}, i}+\operatorname{dist}\left(\mathbf{C}_{\left(k^{\prime}, i\right)}, \mathbf{C}_{(k, i+1)}\right)\right\}
$$

that is applied sequentially starting from the second layer, i.e. $i=1,2, \ldots n-1$. Finally, after selection of the minimum length $d_{k, i+1}$ corresponding to the final layer and applying the backtracking, one can get the desired optimal path in graph. It is described by the recorded indices $\left\{k_{1}, k_{2}, \ldots k_{n-1}, k_{n}\right\}$.
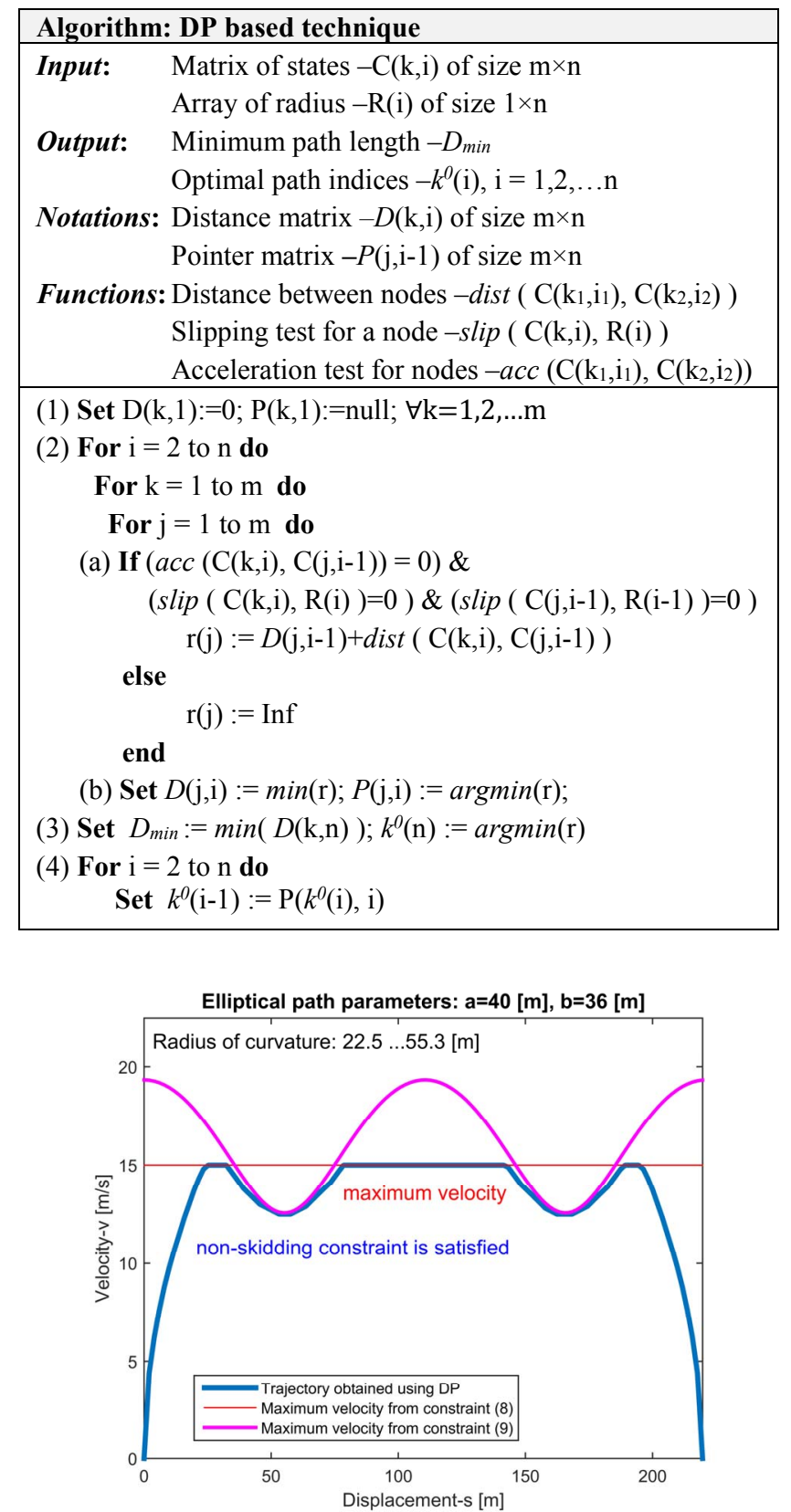

Fig. 3 Motion generated for elliptical path with different curvatures using discrete dynamic programming technique

In more details, an outline of the developed algorithm is presented below in the form of pseudo-codes. The input includes the state matrix $\{C(k, i) \mid k=1,2, \ldots m ; i=1,2, \ldots n\}$ containing the information of displacement and velocity, and also the array of path curvatures $\{R(i) \mid i=1,2, \ldots n\}$. The algorithm operates with two tables $D(k, i)$ and $P(k, i)$ that include the minimum distances for the sub-problem of lower size (for the path $1 \rightarrow \mathrm{i}$ ) and the pointers to the previous locations respectively. The procedure is composed of four basic steps. The first step initializes the distance and pointer matrices. In step (2), the recursive formula (16) is implemented. The computing starts from the second layer, and it tries all possible connections between the nodes in the current layer and the previous one. It includes verifications of the non-skidding constraint and acceleration limit in the substep (2a). The sub-step (2b) finds the minimum path from the current node $C(k, i)$ to the first layer $\{C(j, 1), \forall j\}$ and records the reference to $\{C(j, i-1), \forall j\}$ in the pointer matrix. In steps (3) and (4), the optimal solution is finally obtained and corresponding path is extracted by means of backtracking. To verify capacity of the proposed algorithm, it was applied to the case (c) of the time-optimal motion generation problem considered in the previous section, where the non-skidding constraint (9) is strong and the phase plane technique produced non-feasible motion (see Fig. 1c). The obtained result is presented in Fig. 3, which shows that the developed technique is able to produce feasible time-optimal trajectories with multiple switching points of different types.

\section{An Application Example}

To evaluate the efficiency of the proposed technique, let us apply it to the generation of time-optimal motions along a starshape path whose curvature varies essentially. This path is presented in Fig. 4 and is described by the following parametric equations

$$
\left\{\begin{array}{l}
x=\left(R_{0}+r \cos (k \varphi)\right) \cdot \cos \varphi \\
y=\left(R_{0}+r \cos (k \varphi)\right) \cdot \sin \varphi
\end{array} ; \quad \varphi \in[0,2 \pi]\right.
$$

where $R_{0}=15 \mathrm{~m}, r=5 \mathrm{~m}$ and $k=5$. The parameters of the mobile robot included in the constraints (8) and (9) are assumed to be as follows: $v_{\max }=3.0 \mathrm{~m} / \mathrm{s}, a_{\max }=0.6 \mathrm{~m} / \mathrm{s}^{2}$, $\mu=0.7$. For this path of the length $s_{\max }=142.9 \mathrm{~m}$, the radius of curvature varies essentially, from $+2.76 \mathrm{~m}$ to the positive infinity and from the negative infinity to $-0.87 \mathrm{~m}$, which makes the non-skidding constraint (9) active at the path segments closest to the center.

For this case study, the search space was discretized with the grid $400 \times 150$ which corresponds to the displacement step $\Delta s \approx 0.31 \mathrm{~m}$ and the velocity step $\Delta v=0.02 \mathrm{~m} / \mathrm{s}$. The total computation time for the time-optimal trajectory generation in Matlab R2014b was $23.7 \mathrm{sec}$ (Intel i5 2.67GHz). It is clear that the computing time can be essentially reduced and adapted to the real-time requirements by using more efficient programming environment and by using shorter trajectory planning interval instead of $\left[0, s_{\max }\right]$. 
The obtained time-optimal trajectory is presented in Fig. 5, which clearly shows that the desired motion is composed of multiple acceleration/deceleration segments separated by the relevant number of the switching points, which separate segments with different active constraints (on the control input, on the speed, or on both of them simultaneously). This result is in good agreement with physical nature of the problem, which requires reducing the speed in advance while approaching the path segments with high curvature in order to ensure pure rolling of the robot wheels. Hence, the proposed approach allows finding time-optimal trajectories for any combination of linear/non-linear constraints describing limitations of the robot driving motors and ensuring non-skidding motions.

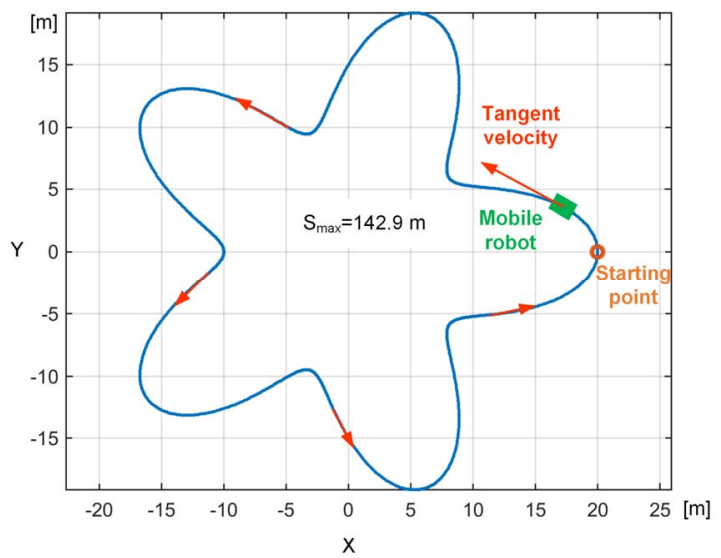

Fig. 4 A star-shape path with variable curvature for mobile robot

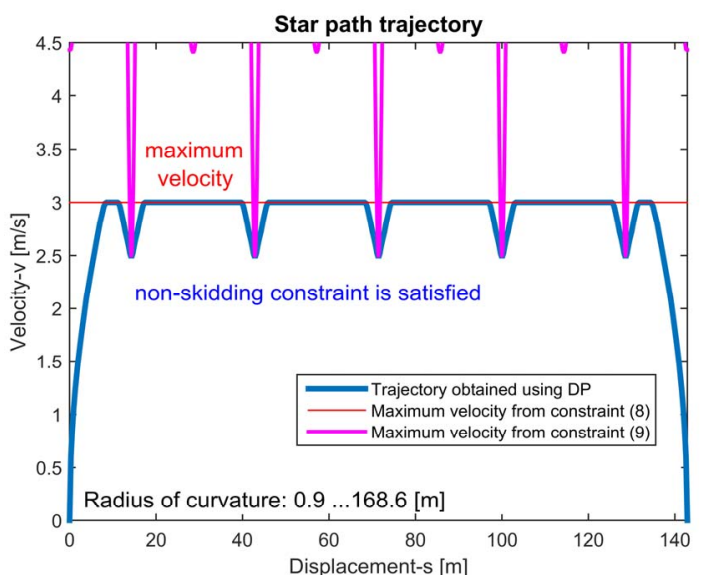

Fig. 5 Time-optimal trajectory for star-shape path ensuring mobile robot motion without skidding (obtained using the DP-based technique)

\section{CONCLUSION}

Current advances in mobile robotics and increasing of achievable motion speeds require essential improvement of existing motion planning algorithms, which in addition to the conventional velocity/acceleration constraints must take into account physical properties of the robot wheels contacts with the driving surface. The latter is also known as the "nonskidding" constraints, which can be hardly integrated into existing motion planning algorithms that are mainly based on the phase-plane technique. To overcome this difficulty, the paper proposes a new method that is able to find time-optimal motions along a curved path that takes into account limitations of the driving motors and also ensures the robot wheels rolling without skidding. After the state space discretization, the original problem is converted into the combinatorial one where the desired robot trajectory is presented as a shortest path on the relevant graph. Dynamic programming is then applied that allows creating a set of feasible trajectories, the best one of which is selected at the final stage. Because of its universal nature, this technique is able to take into account all considered constraints in a similar way and to generate optimal trajectories of complex structure that include multiple switching points. Advantages of this method and its suitability of real-time control were confirmed by several examples dealing the mobile robot motion along the curved paths of elliptic-/star- shape.

\section{REFERENCES}

[1] J. E. Bobrow, S. Dubowsky, and J. Gibson, "Time-optimal control of robotic manipulators along specified paths," The international journal of robotics research, vol. 4, no. 3, pp. 3-17, 1985.

[2] F. Pfeiffer and R. Johanni, "A concept for manipulator trajectory planning," IEEE Journal on Robotics and Automation, vol. 3, no. 2, pp. 115-123, 1987.

[3] Z. Shiller and H.-H. Lu, "Robust computation of path constrained time optimal motions," in Robotics and Automation, 1990. Proceedings., 1990 IEEE International Conference on, 1990, pp. 144-149: IEEE.

[4] Z. Shiller and H.-H. Lu, "Computation of path constrained time optimal motions with dynamic singularities," Journal of dynamic systems, measurement, and control, vol. 114, no. 1, pp. 34-40, 1992.

[5] K. Shin and N. McKay, "Minimum-time control of robotic manipulators with geometric path constraints," IEEE Transactions on Automatic Control, vol. 30, no. 6, pp. 531-541, 1985.

[6] J.-J. Slotine and H. S. Yang, "Improving the efficiency of time-optimal path-following algorithms," IEEE Transactions on Robotics and Automation, vol. 5, no. 1, pp. 118-124, 1989.

[7] H. S. Yang and J.-J. E. Slotine, "Fast Algorithms for Near-MinimumTime Control of Robot Manipulators: Communication," The International journal of robotics research, vol.13, no.6, pp.521-532, 1994.

[8] S. D. Timar, R. T. Farouki, T. S. Smith, and C. L. Boyadjieff, "Algorithms for time-optimal control of CNC machines along curved tool paths," Robotics and Computer-Integrated Manufacturing, vol. 21, no. 1, pp. 37-53, 2005.

[9] H. Dong, M. Cong, D. Liu, and G. Wang, "An effective technique to find a robot joint trajectory of minimum global jerk and distance," in Information and Automation, 2015 IEEE International Conference on, 2015, pp. 1327-1330: IEEE.

[10] J. Dong, P. Ferreira, and J. Stori, "Feed-rate optimization with jerk constraints for generating minimum-time trajectories," International Journal of Machine Tools and Manufacture, vol. 47, no. 12-13, pp. 1941-1955, 2007.

[11] P. Jacobs and J. Canny, "Planning smooth paths for mobile robots," in Nonholonomic Motion Planning: Springer, 1993, pp. 271-342.

[12] P. Soueres and J.-P. Laumond, "Shortest paths synthesis for a car-like robot," IEEE Transactions on Automatic Control, vol. 41, no. 5, pp. 672-688, 1996.

[13] W. Weiguo, C. Huitang, and W. Peng-Yung, "Optimal motion planning for a wheeled mobile robot," in Robotics and Automation, 1999. Proceedings. 1999 IEEE International Conference on, 1999, vol. 1, pp. 41-46: IEEE.

[14] T. Petrinić, M. Brezak, and I. Petrović, "Time-optimal velocity planning along predefined path for static formations of mobile robots," International Journal of Control, Automation and Systems, journal article vol. 15, no. 1, pp. 293-302, February 012017.

[15] J. Gao, A. Pashkevich, and S. Caro, "Manipulator Motion Planning in Redundant Robotic System for Fiber Placement Process," in New Trends in Mechanism and Machine Science: Springer, 2017, pp. 243-252. 